\title{
Epidemiological and clinical features of coronavirus disease 2019 (COVID-19) cases in Guizhou Province in Southwest China
}

Qian Zhang ${ }^{1, *}$, Hong Peng ${ }^{1, *}$, Xinhua Luo ${ }^{1, \#}$, Xiangyan Zhang, ${ }^{2, \#}$, Yan Luo ${ }^{1}$, Xianwei Ye $^{2}$, Rongpin Wang ${ }^{3}$, Hejun Shao ${ }^{1}$, Shanshan Rao' ${ }^{2}$,ing Zhang ${ }^{1}$

${ }^{1}$ Department of Infectious Diseases, Guizhou Provincial People's Hospital, Guiyang, Guizhou 550002, China

${ }^{2}$ Department of Respiratory and Critical Care Medicine, Guizhou Provincial People's Hospital, Guiyang, Guizhou 550002, China

${ }^{3}$ Department of Radiology, Guizhou Provincial People's Hospital, Guiyang, Guizhou 550002, China

* These authors contributed equally to this work.

* Correspondence: Xiangyan Zhang or Xinhua Luo

E-mail: zxy35762@126.com or Luoxinhua1972@126.com

Received: May 08, 2020

Published: August 16, 2020

\begin{abstract}
Most of the clinical research on coronavirus disease 2019 (COVID-2019) has focused on patients in Wuhan, Hubei, China. This study describes the clinical characteristics of COVID-19 patients outside of Hubei. Eighteen confirmed COVID-19 patients at Guizhou Provincial People's Hospital were included. They were divided into nonsevere and severe groups, and their epidemiological characteristics, demographics, clinical manifestations, and laboratory results were reviewed and compared. All patients were infected through human-to-human transmission, and 9 were imported cases. The clinical features were mainly cough (16 [89\%]), fever (11 [61\%]), and sputum production $(9[\mathbf{5 0} \%])$. Most patients had bilateral pneumonia. There were significant differences in the level of lactate dehydrogenase (LDH) $(179$ [142-193] vs 289 [260.25-368.75]), level of serum ferritin (267.2 [100.5-675.2] vs 614.85 [528.5-870.78]), lymphocyte count (1.52 [1.40-1.60] vs 0.59 [0.47- 1.05]), and CD cell count (1576 [1040-1696] vs 444 [312-592] CD3+ T cells, 692 [420-708] vs 144 [128-320] CD4+ T cells, 796 [632- 892] vs 228 [152-284] CD8+ $T$ cells) between the nonsevere group and the severe group $(P<0.05)$. As of Feb. 11 th, 9 people had been discharged after treatment. This study suggests that COVID-19 cases often occur in clusters. Patients with severe COVID-19 had abnormalities in inflammation and immune indicators. Most patients outside Hubei had a good prognosis.
\end{abstract}

Keywords: COVID-19, epidemiological, clinical features, Guizhou

Abbreviations: COVID-19, coronavirus disease 2019; SARS-CoV-2, Severe acute respiratory syndrome coronavirus 2; SARS-CoV, Severe acute respiratory syndrome coronavirus; MERS-CoV, Middle East respiratory syndrome coronavirus; ALI, Acute lung injury; ARDS, Acute respiratory distress syndrome; CT, Computed tomography; M, Male; F, Female; $\mathrm{PaO} 2$, Arterial oxygen pressure; FIO2, Inspiratory oxygen fraction; PCT, Procalcitonin; ESR, Erythrocyte sedimentation rate; CRP, C-reactive protein; LDH, Lactate dehydrogenase; CDC, Centers for Disease Control.

To cite this article: Qian Zhang, et al. Epidemiological and clinical features of coronavirus disease 2019 (COVID-19) cases in Guizhou Province in Southwest China. Inflamm Cell Signal 2020; 7: e1150. doi: 10.14800/ics.1150.

\section{Introduction}

The Chinese Centers for Disease Control and Prevention reported an outbreak of cases of pneumonia in the Huanan Seafood Market, Wuhan city, Hubei Province, on December $31,2019^{[1]}$. The pneumonia cases were found to be caused by a new strain of coronavirus (SARS-CoV-2); this etiology was confirmed by the Ministry of Public Health of China on January 7 th, $2020^{[2]}$. The cases were first reported in the areas surrounding the Huanan Seafood Market, and according to current epidemiological data, SARS-CoV-2 is transmitted between people ${ }^{[3-6]}$. As of March 10, more than 110 thousand cases had been reported globally, with more than 4000 deaths 
have been reported. Cases of infection have spread all over the world ${ }^{[7]}$. The number of confirmed cases of coronavirus disease 2019 (COVID-19) has exceeded that of severe acute respiratory syndrome (SARS) in mainland China in 2002 and 2003 and that of Middle East respiratory syndrome (MERS) in $2012^{[8,9]}$.

Some researchers found that the virus might have been transmitted from bats to humans through an intermediate host ${ }^{[10]}$. Infection with SARS-CoV-2 has several clinical manifestations in humans. The majority of the infected patients develop moderately severe clinical disease, while a certain number of patients develop severe disease characterized by acute lung injury (ALI) and acute respiratory distress syndrome (ARDS) ${ }^{[11,12]}$. The clinical features vary from mild to moderate symptoms to severe symptoms; the former are common in patients under 18 years of age, while the latter are more commonly observed in the elderly population $^{[5,11,12]}$. Patients who have preexisting chronic diseases such as diabetes, obesity, cardiac failure and kidney failure are more likely to develop severe COVID-19 ${ }^{[5,13,14]}$. The current studies have mainly been based on case studies in Wuhan city, and there have been few studies on the situation in Guizhou Province, as it is one of the provinces with the fewest infected patients in Southwest China. This article describes the epidemiological features of COVID-19 patients treated in Guizhou Provincial People's Hospital and the clinical features, countermeasures and therapeutic outcomes in patients with nonsevere and severe COVID-19. The aim is to provide additional information to improve the understanding of the COVID-19 pandemic.

\section{Materials and Methods}

This was a retrospective, single-center study. Data were obtained from confirmed COVID-19 patients admitted to Guizhou Provincial People's Hospital from January 29th, 2020, to February 11th, 2020. The diagnosis of COVID-19 was confirmed in all study patients by two positive nucleic acid tests. All the data of the included cases were shared with the WHO. The study was approved by Guizhou Provincial People's Hospital Ethics Committee. The need to obtain informed consent was waived because of the retrospective nature of the study and the use of anonymous clinical data. We obtained the epidemiological, demographic, clinical, laboratory and radiological characteristics; treatment information; and outcome data from patients' medical records. Clinical outcomes were followed up to February 15th, 2020. All data were checked by two physicians (ZQ and LSY). Patients with suspected cases of COVID-19 were admitted and quarantined, and throat swab, alveolar lavage fluid, stool and breastmilk samples were collected and sent to the Chinese Centers for Disease Control and Prevention for the detection of SARS-CoV-2 with a quantitative polymerase chain reaction assay. Sputum or endotracheal aspirates were obtained at admission for the identification of pathogenic bacteria or fungi. Additionally, all patients underwent chest CT.

The epidemiological data (i.e., history of travel to Wuhan or close contact with confirmed patients); demographics; signs and symptoms on admission; comorbidities; laboratory results; chest CT findings; treatment received for COVID-19; and clinical outcomes were analyzed.

\section{Statistical Analysis}

Continuous variables are expressed as the medians (IQRs) and were compared with the Mann-Whitney $U$ test; categorical variables are expressed as numbers $(\%)$ and were compared by $\chi^{2}$ tests between the severe and nonsevere groups. A P value less than 0.05 was considered statistically significant. Statistical analyses were conducted using SPSS software, version 25.0.

\section{Results}

By February 11, 2020, 18 admitted hospital patients had been identified as having laboratory-confirmed COVID-19 in Guiyang. Among these patients, 9 had a history of travel to epidemic areas, and 8 out of those 9 patients were local citizens who had returned home from Hubei Province, including a household of 3 family members, whereas the other patient was a citizen of Wuhan city. Two of the 9 imported patients were a 50-day-old infant and a 4-year-old child. The other 9 patients were infected via close contact with individuals with confirmed COVID-19. Three of the 9 were infected while sharing meals with family members (Figure 1). Family gatherings were the source of infection among most of the patients. The average incubation period was generally 11.5 (5.5-14) days.

Of the 18 patients, 7 (38.9\%) were diagnosed with severe COVID-19 because their arterial oxygen tension $\left(\mathrm{PaO}_{2}\right)$ over inspiratory oxygen fraction $\left(\mathrm{FIO}_{2}\right)$ was less than $300 \mathrm{mmHg}$ or their oxygen saturation level was less than $93 \%$. Only 3 severe COVID-19 patients had a history of travel to an epidemic area. The median age of all the patients was 40.5 (23-55) years (34 [15-58] years in the nonsevere group vs 44 [37-54] years in the severe group [P=0.007]). Two children and two elderly people were in the nonsevere group, while the age of patients in critical condition ranged from 19 to 60 years. Eight (44\%) of the infected patients were men; less than half had underlying diseases (7 [39\%]), including hypertension (5 [28\%]), diabetes (1 [5.5\%]), and pulmonary disease (1 [5.5\%]). There were no significant differences between the two groups of patients with regard to comorbidities. The main symptoms at the onset of illness were cough (16 [89\%]), fever (11 [61\%]), 

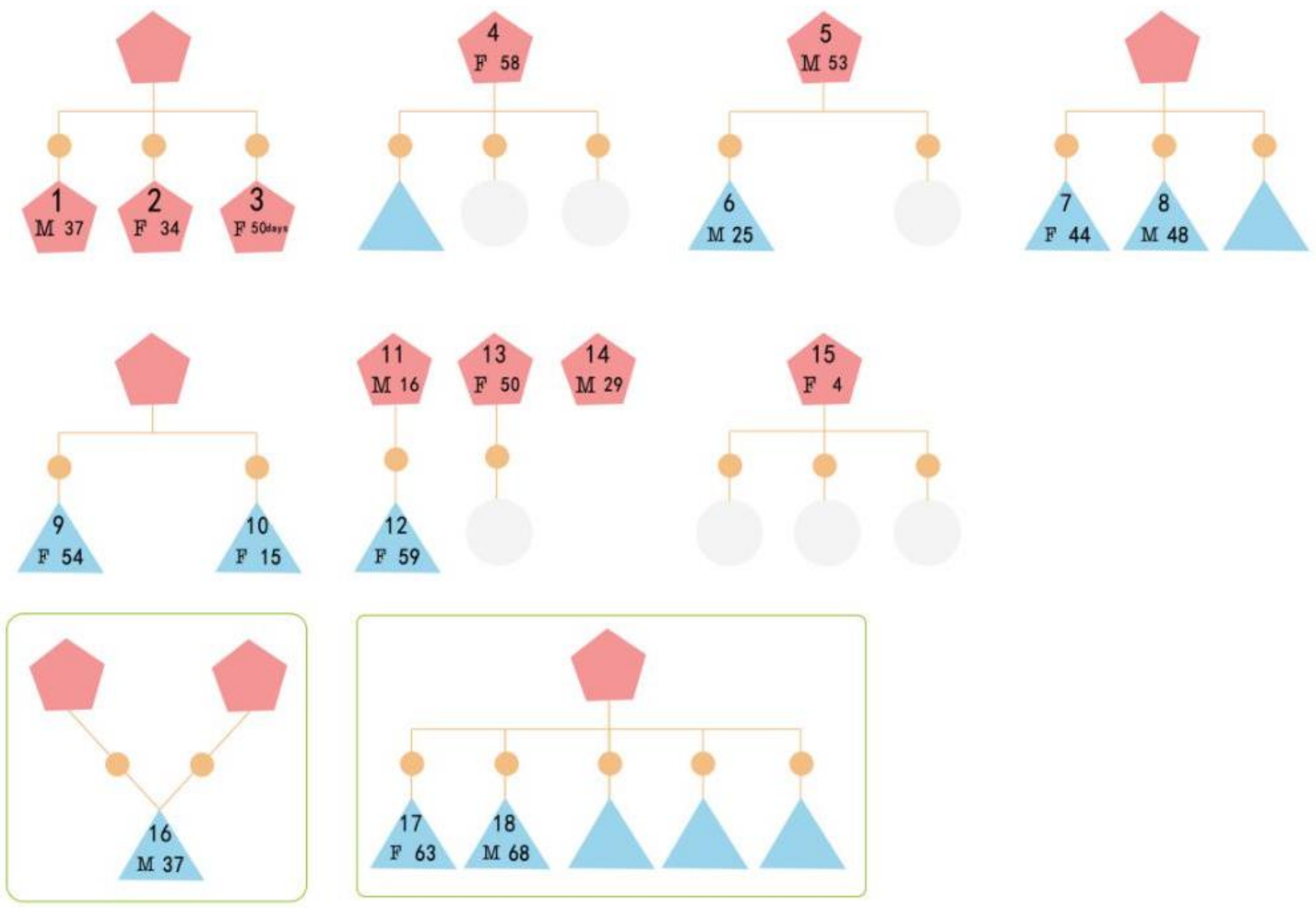

Figure 1. Sources of infection for 18 COVID-19 patients. (M=male, $\mathrm{F}=$ female)

and sputum production (9 [50\%]); other symptoms were myalgia or fatigue (4 [22\%]), shortness of breath (4 [22\%]), chills $(2[11 \%])$, nausea and vomiting (2[11\%]), sore throat (1 [5.6\%]), dizziness (1 [5.6\%]) and rhinorrhea (1 [5.6\%]). In the severe group, all the patients $(100 \%)$ had fever, while 4 (36\%) patients in the nonsevere group had fever $(\mathrm{P}=0.013)$ (Table 1).

The neutrophil counts in the two groups remained normal; however, the lymphocyte count in the severe group was 0.59 (0.47-1.05), which was obviously lower than the normal range, whereas the lymphocyte count in the nonsevere group was 1.52 (1.40-1.60). There was a significant difference $(\mathrm{P}=0.016)$ between the two groups. The monocyte and blood platelet counts, as well as the coagulation function and hepatorenal function, remained normal. No differences were observed between the two groups. However, the LDH levels were elevated to varying degrees in all patients, and the levels were particularly elevated in the severe group (179 [142-193] in the nonsevere group vs 289 [260.25-368.75] in the severe group) $(\mathrm{P}=0.027)$. The levels of PCT, ESR, serum ferritin, and Creactive protein were also elevated in the severe group, and there was a significant difference in the serum ferritin levels between the nonsevere and severe groups (267.2 [100.5-675.2] in the nonsevere group vs 614.85 [528.5-870.78] in the severe group) ( $\mathrm{P}=0.028)$. The $\mathrm{CD} 3+\mathrm{T}$ cell $(1576$ [1040-1696] in the nonsevere group vs 444 [312-592] in the severe group), CD4+ T cell (692 [420-708] in the nonsevere group vs 144 [128-320] in the severe group) and CD8+ T cell (796 [632892] in the nonsevere group vs 228 [152-284] in the severe group) counts were lower than normal ranges in the severe group, and the counts were significantly different between the severe and nonsevere groups $(\mathrm{P}<0.000)$. Because the results 
http://www.smartscitech.com/index.php/ics

Table 1. Demographics and baseline characteristics of COVID-19 patients.

\begin{tabular}{|c|c|c|c|c|}
\hline Characteristics & All patients $(n=18)$ & $\begin{array}{c}\text { Nonsevere group } \\
(n=11)\end{array}$ & $\begin{array}{c}\text { Severe group } \\
(n=7)\end{array}$ & P Value \\
\hline Age, years\# & $40.5(23-55)$ & $34(15-58)$ & $44(37-54)$ & 0.007 \\
\hline$\leq 7$ & $2(11 \%)$ & $2(18 \%)$ & 0 & 0.497 \\
\hline $7-18$ & $2(11 \%)$ & $2(18 \%)$ & 0 & 0.497 \\
\hline $19-60$ & $12(67 \%)$ & $5(45 \%)$ & $7(100 \%)$ & 0.038 \\
\hline$\geq 60$ & $2(11 \%)$ & $2(18 \%)$ & 0 & 0.497 \\
\hline \multicolumn{5}{|l|}{ Sex } \\
\hline Men & $8(44 \%)$ & $4(36 \%)$ & $4(57 \%)$ & 0.63 \\
\hline Women & $10(56 \%)$ & $7(64 \%)$ & $3(43 \%)$ & 0.63 \\
\hline $\begin{array}{c}\text { History of travel to an epidemic } \\
\text { area }\end{array}$ & $9(50 \%)$ & $6(54.5 \%)$ & $3(42.9 \%)$ & 0.5 \\
\hline $\begin{array}{l}\text { Close-contact with individuals with } \\
\text { confirmed COVID-19 }\end{array}$ & $9(50 \%)$ & $5(45.5 \%)$ & $4(57.1 \%)$ & 0.5 \\
\hline Any comorbidity & $7(39 \%)$ & $4(36 \%)$ & $3(43 \%)$ & 0.583 \\
\hline Hypertension & $5(28 \%)$ & $3(17 \%)$ & $2(29 \%)$ & 0.676 \\
\hline Diabetes & $1(5.5 \%)$ & $1(5.6 \%)$ & 0 & 0.611 \\
\hline Pulmonary disease & $1(5.5 \%)$ & 0 & $1(14 \%)$ & 0.389 \\
\hline \multicolumn{5}{|l|}{ Signs and symptoms at admission } \\
\hline Cough & $16(89 \%)$ & $10(91 \%)$ & $6(86 \%)$ & 0.641 \\
\hline Fever\# & $11(61 \%)$ & $4(36 \%)$ & $7(100 \%)$ & 0.013 \\
\hline Sputum production & $9(50 \%)$ & $4(36 \%)$ & $5(71 \%)$ & 0.335 \\
\hline Shortness of breath & $4(22 \%)$ & $1(9 \%)$ & $3(43 \%)$ & 0.245 \\
\hline Myalgia or fatigue & $4(22 \%)$ & $1(9 \%)$ & $3(43 \%)$ & 0.245 \\
\hline Nausea and vomiting & $2(11 \%)$ & $1(9 \%)$ & $1(14 \%)$ & 0.641 \\
\hline Chills & $2(11 \%)$ & $1(9 \%)$ & $1(14 \%)$ & 0.641 \\
\hline Sore throat & $1(5.6 \%)$ & $1(9 \%)$ & 0 & 0.611 \\
\hline Dizziness & $1(5.6 \%)$ & 0 & $1(14 \%)$ & 0.389 \\
\hline Diarrhea & $1(5.6 \%)$ & 0 & $1(14 \%)$ & 0.389 \\
\hline Rhinorrhea & $1(5.6 \%)$ & $1(9 \%)$ & 0 & 0.611 \\
\hline More than one sign or symptom & $14(78 \%)$ & $7(64 \%)$ & $7(100 \%)$ & 0.119 \\
\hline
\end{tabular}

\#: The value in the two groups was considered statistically significant.

of the blood tests were substantially different between children and adults, the analysis excluded the two young children. No abnormalities were observed on chest $\mathrm{CT}$ in 2 of the patients, while patchy inflammatory exudation was observed in the other patients. Nine (39\%) patients had bilateral pneumonia, and $1(5.6 \%)$ patient had unilateral pneumonia. Eight (44\%) patients had multiple areas of mottling and ground glass opacity (Figure 2). In the severe group, most patients had multiple areas of mottling and ground glass opacity (5 [71.4\%]). Nucleic acid testing of the throat swab was negative, while nucleic acid testing of bronchoalveolar lavage fluid was positive in one patient with severe COVID-19. Two patients in the nonsevere group had positive results for nucleic acid in feces samples. One lactating female patient was identified as being infected on the same day as was her 50-day-old daughter. The nucleic acid testing of her breast milk was negative (Table 2). All the bacteria and fungi cultures from the patients were also negative.

All patients were isolated in the hospital. All of them received antiviral treatment, including oseltamivir $(75 \mathrm{mg}$ every 12 h, orally), Arbidol (200 mg every 8 h, orally), or lopinavir and ritonavir tablets (500 $\mathrm{mg}$ twice daily, orally). Most patients (17 [94.4\%]) were given antibiotic treatment; 7 (38.9\%) patients were given antifungal therapy. Six (85.7\%) of the patients in the severe group used corticosteroids, while $2(18.2 \%)$ of the patients in the nonsevere group used 

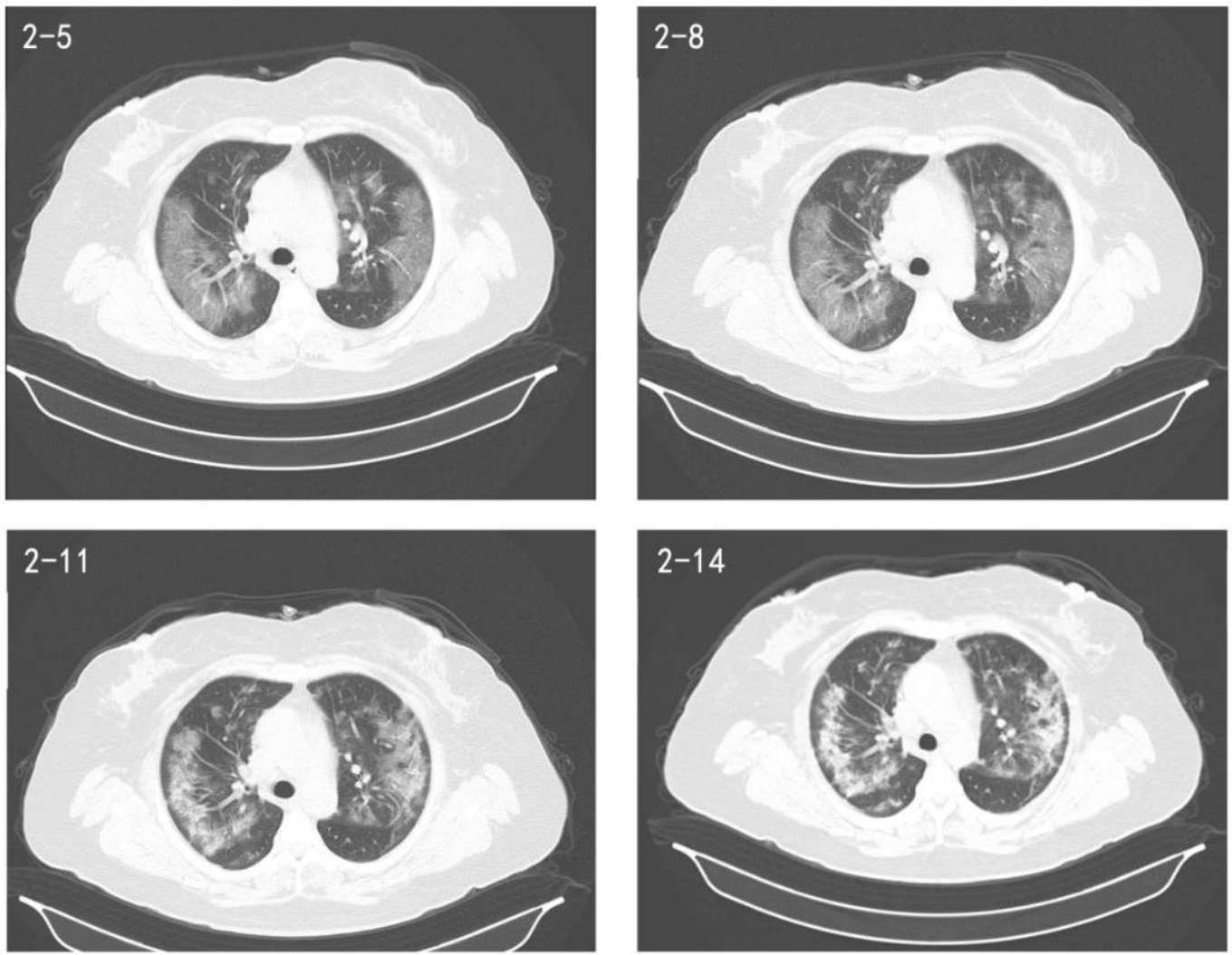

Figure 2. Chest CT images. These chest CT images were obtained from a 54-year-old female patient. On February 5, large ground glass opacities were observed on both sides of the lung fields, mostly beneath the pleura. On February 8, the lesion area and density had increased. On February 11 , the lesion area had increased, and consolidation was observed. The ground glass opacity in the upper right lung started to be assimilated. By February 14, more of the lesion area had been assimilated, and the rest had developed into fibrosis.

corticosteroids $(\mathrm{P}=0.013)$. Twelve $(66.7 \%)$ patients required oxygen therapy, of whom 3 (42.9\%) patients in the severe group required noninvasive ventilation or high-flow nasal cannula. Most of the patients were given drugs to improve their immunity. Targeted traditional Chinese medicine was also administered to all the patients. By February 15, 2020, 9 ( 5 in the nonsevere group, 4 in the severe group) patients had been discharged from the hospital (Table 3). Their average period of treatment in the hospital was 10 (5.5-13.5) days.

\section{Discussion}

This essay is a cross-sectional study of the COVID-19 cases in Guizhou Province, the least affected region in the Southwest China. Eighteen patients with confirmed cases treated in Guizhou Province People's Hospital were investigated. The epidemiological, demographic, and clinical characteristics; laboratory test results; treatments; and prognoses were compared between the patients in the nonsevere and severe groups.

We find that the patients are identified as the second and third generation patients. Half of them had a history of travel to an epidemic area and were infected at family gatherings. The COVID-19 outbreak started during the Chinese Spring Festival. A large number of migrant workers traveled to gather with family members. Thus, the location and the timing were critical factors that made it difficult to prevent the spread of the infection. The incubation period of the disease was 11.5 days in this study, which is similar to the 14-day incubation period reported previously ${ }^{[5]}$. Moreover, most of the patients were between the ages of 19 and 60 years. This group is more likely to be infected because they are more likely to travel within and between regions than children and seniors. We also found no obvious predominance of one sex among the patients, which conflicts with the reports that stated that males are more likely to be infected than females ${ }^{[5,15]}$. Therefore, future investigations should have larger sample sizes. Patients with other chronic diseases accounted for a small proportion of the whole sample of patients in this study, and there were slight differences among the patients before they developed either nonsevere or severe COVID-19. Therefore, in addition to preexisting chronic diseases, other factors, such as immunity 
http://www.smartscitech.com/index.php/ics

Table 2. Laboratory results of COVID-19 patients.

\begin{tabular}{|c|c|c|c|c|c|}
\hline & Normal range & $\begin{array}{c}\text { All patients } \\
(\mathrm{n}=18)\end{array}$ & $\begin{array}{c}\text { Nonsevere group } \\
(\mathbf{n}=11)\end{array}$ & $\begin{array}{c}\text { Severe group } \\
(n=7)\end{array}$ & $\begin{array}{c}\mathbf{P} \\
\text { Value } \\
\end{array}$ \\
\hline White blood cell count, $\times 10^{9} / \mathrm{L}$ & $3.5-9.5$ & $4.35(3.29-6.65)$ & $4.35(3.59-6.23)$ & $4.78(2.70-8.11)$ & 0.96 \\
\hline Neutrophil count, $\times 10^{9} / \mathrm{L}$ & $1.8-6.3$ & $2.46(1.87-4.58)$ & $2.31(1.66-4.21)$ & $2.79(1.99-7.22)$ & 0.5 \\
\hline Lymphocyte count, $\times 10^{9} / \mathrm{L} \#$ & $1.1-3.2$ & $1.41(0.59-1.56)$ & $1.52(1.40-1.60)$ & $0.59(0.47-1.05)$ & 0.016 \\
\hline Monocyte count, $\times 10^{9} / \mathrm{L}$ & $0.1-0.6$ & $0.37(0.3-0.53)$ & $0.41(0.36-0.57)$ & $0.27(0.12-0.39)$ & 0.097 \\
\hline Platelet count, $\times 10^{9} / \mathrm{L}$ & $125-350$ & $229(189-290)$ & $218(177-304)$ & $246.5(185.5-286)$ & 0.84 \\
\hline Prothrombin time, $\mathrm{s}$ & $9.2-12.2$ & $10.6(10.2-11.3)$ & $10.5(10.3-11.3)$ & $10.8(10-11.3)$ & 0.98 \\
\hline Activated partial thromboplastin time, $\mathrm{s}$ & $21.1-36.5$ & $26.1(24.2-28.9)$ & $28.1(24.2-30.0)$ & $25.2(24.2-27.9)$ & 0.432 \\
\hline D-dimer, mg/L & $0-1.5$ & $0.43(0.22-0.62)$ & $0.34(0.19-0.55)$ & $0.51(0.38-0.76)$ & 0.139 \\
\hline Albumin, $\mathrm{g} / \mathrm{L}$ & $40-55$ & $40.3(37.4-41.7)$ & $40.3(37.4-43.5)$ & $39.25(36.1-40.7)$ & 0.24 \\
\hline Alanine aminotransferase, U/L & $9-50$ & $31(12-43.5)$ & $15(11-42)$ & $33(17.3-55.3)$ & 0.287 \\
\hline Aspartate aminotransferase, U/L & $15-40$ & $25(14.5-35)$ & $15(13-32)$ & $27.5(24.8-49.3)$ & 0.086 \\
\hline Total bilirubin, $\mathrm{mmol} / \mathrm{L}$ & $3.4-20.5$ & $15.1(12.1-18.4)$ & $12.7(9.6-18.2)$ & $16.85(13.9-19.5)$ & 0.312 \\
\hline Creatine kinase, U/L & $50-310$ & $70(48.3-190.8)$ & $49(40-107)$ & $121(64-287.5)$ & 0.261 \\
\hline Creatinine, $\mu \mathrm{mol} / \mathrm{L}$ & $57-97$ & $62(56.5-76.5)$ & $72(57-80)$ & $60(55.8-82.3)$ & 0.887 \\
\hline Blood urea nitrogen, $\mathrm{mmol} / \mathrm{L}$ & $3.1-8$ & $3.91(3.3-6)$ & $3.91(2.9-4.6)$ & $4.65(3.3-6.7)$ & 0.233 \\
\hline Lactate dehydrogenase, U/L\# & $120-250$ & $231(175.5-313)$ & $179(142-193)$ & $289(260.3-368.8)$ & 0.027 \\
\hline Procalcitonin, ng/mL & $0-0.046$ & $0.04(0.02-0.07)$ & $0.03(0.02-0.06)$ & $0.065(0.04-0.09)$ & 0.582 \\
\hline Interleukin-6, pg/mL & $0-7$ & $2.95(1.5-6.89)$ & $2.95(1.5-5.97)$ & $3.9(1.5-22.67)$ & 0.888 \\
\hline Erythrocyte sedimentation rate $\mathrm{mm} / \mathrm{h}$; & $2-20.9$ & $42(19-63)$ & $41(10-66)$ & $45(33.3-63.5)$ & 0.689 \\
\hline Serum ferritin, ng/mL\# & $30-400$ & $\begin{array}{c}548.8(192.3- \\
682.8)\end{array}$ & $267.2(100.5-675.2)$ & $614.9(528.5-870.8)$ & 0.028 \\
\hline C-reactive protein, $\mathrm{mg} / \mathrm{L}$ & $0-5$ & $7.16(1.65-21.91)$ & $2.61(1.13-15.53)$ & $14.57(5.53-34.51)$ & 0.642 \\
\hline CD3+T cell count\# & $770-2860$ & $1576(792-2260)$ & $1576(1040-1696)$ & $444(312-592)$ & 0.000 \\
\hline CD4+T cell count\# & $500-1440$ & $692(384-924)$ & $692(420-708)$ & $144(128-320)$ & 0.000 \\
\hline CD8+T cell count \# & $238-1250$ & $796(540-892)$ & $796(632-892)$ & $228(152-284)$ & 0.000 \\
\hline CD4:CD8 & $1-2.47$ & $1.28(0.83-1.50)$ & $1.01(0.78-2.31)$ & $1.37(0.66-1.42)$ & 0.931 \\
\hline \multicolumn{6}{|l|}{ Chest CT findings } \\
\hline Normal & & $2(11 \%)$ & $2(18 \%)$ & 0 & 0.497 \\
\hline Unilateral pneumonia & & $1(5.6 \%)$ & $1(9 \%)$ & 0 & 0.611 \\
\hline Bilateral pneumonia & & $7(39 \%)$ & $5(45 \%)$ & $2(28.6 \%)$ & 0.637 \\
\hline Multiple mottling and ground-glass opacity & & $8(44 \%)$ & $3(27 \%)$ & $5(71.4 \%)$ & 0.145 \\
\hline \multicolumn{6}{|l|}{ RT-PCR testing for SARS-CoV-2 } \\
\hline Oropharyngeal swab & & $17(94.4 \%)$ & $11(100 \%)$ & $6(85.7 \%)$ & NA \\
\hline Stool & & $2(11 \%)$ & $2(18.2 \%)$ & 0 & NA \\
\hline Alveolar lavage & & $1(5.6 \%)$ & 0 & $1(14.3 \%)$ & NA \\
\hline Breastmilk & & 0 & 0 & 0 & NA \\
\hline
\end{tabular}

\#: The value in the two groups was considered statistically significant.

and inflammation, should be examined when conducting research on the development of severe COVID-19. In this cohort, the main symptoms were cough, fever, and sputum production. Bilateral ground glass opacities were observed on chest CT. Similar symptoms and CT results were observed in SARS and MERS patients ${ }^{[13,16]}$. A few patients had digestive symptoms, including nausea, vomiting and diarrhea, which were also seen in MERS and SARS patients. However, the 
http://www.smartscitech.com/index.php/ics

Table 3. Treatments and outcomes of COVID-19 patients.

\begin{tabular}{|c|c|c|c|c|}
\hline & $\begin{array}{c}\text { All patients } \\
(n=18)\end{array}$ & $\begin{array}{c}\text { Nonsevere group } \\
(n=11)\end{array}$ & $\begin{array}{c}\text { Severe group } \\
(n=7)\end{array}$ & P Value \\
\hline \multicolumn{5}{|l|}{ Treatment } \\
\hline Antiviral therapy & $18(100 \%)$ & $11(100 \%)$ & $7(100 \%)$ & NA \\
\hline Antibiotic therapy & $17(94.4 \%)$ & $10(90.9 \%)$ & $7(100 \%)$ & 0.611 \\
\hline Antifungal therapy & $7(38.9 \%)$ & $3(7.2 \%)$ & $4(57.1 \%)$ & 0.332 \\
\hline Use of corticosteroids\# & $8(44.4 \%)$ & $2(18.2 \%)$ & $6(85.7 \%)$ & 0.013 \\
\hline \multicolumn{5}{|l|}{ Oxygen support } \\
\hline Nasal cannula & $12(66.7 \%)$ & $5(45.5 \%)$ & $7(100 \%)$ & 0.038 \\
\hline Noninvasive ventilation or high-flow nasal cannula\# & $3(16.7 \%)$ & 0 & $3(42.9 \%)$ & 0.043 \\
\hline Enhanced immunotherapy & $12(66.7 \%)$ & $6(54.5 \%)$ & $6(85.7 \%)$ & 0.316 \\
\hline Chinese medicine therapy & $18(100 \%)$ & $11(100 \%)$ & $7(100 \%)$ & NA \\
\hline \multicolumn{5}{|l|}{ Clinical outcome } \\
\hline Remained in hospital & $9(50 \%)$ & $6(54.5 \%)$ & $3(42.9 \%)$ & 0.5 \\
\hline Discharged & $9(50 \%)$ & $5(45.5 \%)$ & $4(57.1 \%)$ & 0.5 \\
\hline Died & 0 & 0 & 0 & NA \\
\hline
\end{tabular}

\#: The value in the two groups was considered statistically significant.

likelihood of developing digestive symptoms is relatively lower in COVID-19 patients $^{[13]}$.

Previous research on SARS-CoV and MERS-CoV showed that humans could be affected by direct and indirect damage. Direct damage refers to the damage inflicted on the target cells infected by virus, while indirect damage is caused by the immune response, circulatory dysfunction and anoxia. It is thought that the pathological effects on cells due to viral infection and the host immunoreactions to viruses are critical in evaluating the severity of the disease ${ }^{[17]}$. SARS-CoV, MERS-CoV and SARS-CoV-2 all cause acute respiratory disease with symptoms such as low-grade fever, cough and ALI, and ARDS or death can develop ${ }^{[18-20]}$. The disease is characterized by a high viral load and symptoms such as fever and cough in the early stage. Then, patients develop symptoms of pneumonia as the viral titer decreases ${ }^{[19]}$. A minority of the patients developed ARDS and multiple organ failure, resulting in death. As the viral titer continuously decreased, the worsening of a patient's condition is thought to be the result of intense immune and inflammatory responses. According to recent cohort studies, the ESR and the levels of serum ferritin, CRP, PCT, and LDH are direct or indirect indicators of the degree of inflammation. The levels of all these indicators were higher in the severe group than in the nonsevere group. The levels of serum ferritin and LDH were substantially higher in the severe group than in the nonsevere group. The inflammatory cytokine storm is a critical factor that results in severe consequences. Moreover, the specificity of the responses of $\mathrm{T}$ cells to the SARS-CoV-2 infection is the key to eliminating the virus and preventing more damage to the host $^{[21,22]}$. Furthermore, $T$ cells can also suppress innate immunity if the response is excessive ${ }^{[23,24]}$. This study showed that the counts of the peripheral blood lymphocytes, CD3+ T cells, CD4+ $\mathrm{T}$ cells and CD8+ $\mathrm{T}$ cells were lower than the normal ranges in the severe group and were significantly different between the severe and the nonsevere groups. This shows that $\mathrm{T}$ cell apoptosis causes lymphocyte exhaustion, and a decline in the peripheral blood lymphocyte count indicates the development of severe disease and possible mortality. T cells, especially CD4+ T cells and CD8+ T cells, combat the viral infection by producing an inflammatory cytokine storm ${ }^{[25]}$. CD4+ $\mathrm{T}$ cells induce the production of specific antibodies against the virus by activating T-dependent B cells. CD8+ T cells, which are cytotoxic agents, kill infected cells. CD8+ T cells account for $80 \%$ of the inflammatory cells infiltrating the pulmonary interstitium and eliminate SARSCoV-2 in the infected cells, which will damage host immunity. CD4+ $\mathrm{T}$ cell exhaustion is related to the reduction in the lymphocyte count in the lung and the generation of neutralizing antibodies and cytokines. Exhaustion delays the elimination of the virus and leads to immune-mediated interstitial pneumonia ${ }^{[26]}$. Based on the positive nucleic acid test results in throat swab, bronchoalveolar lavage fluid and feces samples, the virus is transmitted through the respiratory and digestive tracts. There is no evidence of transmission via breast milk, as the nucleic acid tests of breast milk were negative.

Prompt treatment is critical in the early stage of COVID-19; 
however, no effective antiviral drugs have been developed for clinical use. Based on the positive clinical effects of lopinavir/ritonavir on SARS and the mildly curative effects of Arbidol in the early stage, the combined use of lopinavir/ritonavir and Arbidol is now the main antivirus treatment ${ }^{[27]}$. While the remdesivir was very effective against MERS-CoV, and one COVID-19 patient obtained a satisfying result after taking remdesivir, remdesivir is still undergoing clinical trials in Wuhan ${ }^{[27-29]}$. The extensive use of remdesivir has not been reported thus far. Other antibacterial and antifungal drugs have been administered to some patients prophylactically. The short-term administration of low doses of glucocorticoids to patients with severe disease is controversial. Some believe that glucocorticoids could delay the elimination of the virus and might not have positive effects on the resolution of pulmonary inflammation ${ }^{[30]}$. For people with low immunity, the use of thymosin has been suggested to reduce the time needed to eliminate the virus and prevent patients from developing more severe disease. The concomitant use of targeted Chinese traditional medicine and Western medicine is encouraged. Noninvasive ventilation treatment is provided for patients with acute respiratory distress.

Meanwhile, this study had two limitations. First, a larger sample size is needed. Given the current situation, this research excluded suspected infections and people under medical observation; therefore, only 18 patients with confirmed cases of COVID-19 in Guizhou Province were included. Second, due to the small sample size, it was difficult to build a model based on the relevant factors to predict the prognosis. However, the data provided in this article could contribute to the understanding and evaluation of the epidemiological and clinical features of patients in places outside of Wuhan.

COVID-19 often develops in family clusters and is transmitted through the respiratory and digestive tracts. Patients with severe COVID-19 have abnormally high levels of inflammation and reduced immunity. More importantly, most of the patients recovered well after receiving appropriate treatment.

\section{Ethics Approval}

The study was approved by the Ethics Committee of the Guizhou Provincial People's Hospital.

\section{Patient Consent for Publication}

Written informed consent was obtained from the patients for the publication of clinical information.

\section{Acknowledgments}

The authors thank the Guizhou CDC for the SARS-CoV-2 nucleic acid tests.

\section{Funding}

This study was supported by the Guizhou Science and Technology Project (QKHZC[2020]4Y002) and the Guiyang Science and Technology Project (ZKXM[2020]4).

\section{Conflicting Interest}

No conflicts of interest exist.

\section{References}

1. Organization WH. Pneumonia of unknown cause Accessed 5 January $2020 \mathrm{https} / / / \mathrm{www}$.who.int/csr/don/05-january2020-pneumonia-of-unkown-cause-china/en/

2. Organization WH. Novel coronavirus. China. Accessed 12 January 2020, https://www.who.int/csr/don/12-january2020-novel-coronavirus-china/en/

3. Prevention CfDCa. 2019 Novel coronavirus, Wuhan, China: 2019-nCoV situation summary. Accessed January 28, 2020 https://www.cdc.gov/coronavirus/2019$\mathrm{nCoV} /$ summary.html

4. Chan JF, Yuan S, Kok KH, et al. A familial cluster of pneumonia associated with the 2019 novel coronavirus indicating person-to-person transmission: a study of a family cluster. Lancet. 2020;395(10223):514-523.

5. Huang C, Wang Y, Li X, et al. Clinical features of patients infected with 2019 novel coronavirus in Wuhan, China. Lancet. 2020;395(10223):497-506.

6. Phan LT, Nguyen TV, Luong QC, et al. Importation and Human-to-Human Transmission of a Novel Coronavirus in Vietnam. N Engl J Med. 2020.[doi:10.1056/ EJMc2001272]

7. Organization WH. Novel Coronavirus (COVID-19). Situation report. Accessed 17 February 2020, https://www.who.int/docs/default-

source/coronaviruse/situation-reports/20200217-sitrep-28covid-19.pdf?sfvrsn=a19cf2ad_2

8. Cheng VC, Lau SK, Woo PC, et al. Severe acute respiratory syndrome coronavirus as an agent of emerging and reemerging infection. Clin Microbiol Rev. 2007;20(4):66094.

9. Chan JF, Lau SK, To KK, et al. Middle East respiratory syndrome coronavirus: another zoonotic betacoronavirus causing SARS-like disease. Clin Microbiol Rev. 2015;28(2):465-522.

10. Chan JF, Kok KH, Zhu Z, et al. Genomic characterization of the 2019 novel human-pathogenic coronavirus isolated from a patient with atypical pneumonia after visiting Wuhan. Emerging microbes \& infections. 2020;9(1):221-236.

11. Leong HN, Chan KP, Oon LL, et al. Clinical and laboratory findings of SARS in Singapore. Annals of the Academy of Medicine, Singapore. 2006;35(5):332-9. 
http://www.smartscitech.com/index.php/ics

12. Arabi YM, Arifi AA, Balkhy HH, et al. Clinical course and outcomes of critically ill patients with Middle East respiratory syndrome coronavirus infection. Annals of internal medicine. 2014;160(6):389-97.

13. Assiri A, Al-Tawfiq JA, Al-Rabeeah AA, et al. Epidemiological, demographic, and clinical characteristics of 47 cases of Middle East respiratory syndrome coronavirus disease from Saudi Arabia: a descriptive study. The Lancet Infectious diseases. 2013;13(9):752-61.

14. Saad M, Omrani AS, Baig K, et al. Clinical aspects and outcomes of 70 patients with Middle East respiratory syndrome coronavirus infection: a single-center experience in Saudi Arabia. International journal of infectious diseases : IJID : official publication of the International Society for Infectious Diseases. 2014;29:301-6.

15. $\mathrm{Xu} \mathrm{XW}, \mathrm{Wu} \mathrm{XX}$, Jiang $\mathrm{XG}$, et al. Clinical findings in a group of patients infected with the 2019 novel coronavirus (SARS-Cov-2) outside of Wuhan, China: retrospective case series. BMJ (Clinical research ed). 2020;368:m606. [doi:10.1136/bmj.m606]

16. Lee N, Hui D, Wu A, et al. A major outbreak of severe acute respiratory syndrome in Hong Kong. $N$ Engl J Med. 2003;348(20):1986-94.

17. Guo Y, Korteweg C, McNutt MA, Gu J. Pathogenetic mechanisms of severe acute respiratory syndrome. Virus research. 2008;133(1):4-12.

18. Kuiken T, Fouchier RA, Schutten M, et al. Newly discovered coronavirus as the primary cause of severe acute respiratory syndrome. Lancet. 2003;362(9380):263-70.

19. Peiris JS, Chu CM, Cheng VC, et al. Clinical progression and viral load in a community outbreak of coronavirusassociated SARS pneumonia: a prospective study. Lancet. 2003;361(9371):1767-72.

20. Peiris JS, Guan Y, Yuen KY. Severe acute respiratory syndrome. Nature medicine. 2004;10(12 Suppl):S88-97.

21. Zhao J, Zhao J, Perlman S. T cell responses are required for protection from clinical disease and for virus clearance in severe acute respiratory syndrome coronavirus-infected mice. Journal of Virology. 2010;84(18):9318-25.

22. Zhao J, Zhao J, Legge K, Perlman S. Age-related increases in $\mathrm{PGD}(2)$ expression impair respiratory DC migration, resulting in diminished $\mathrm{T}$ cell responses upon respiratory virus infection in mice. The Journal of clinical investigation. 2011;121(12):4921-30.

23. Kim KD, Zhao J, Auh S, et al. Adaptive immune cells temper initial innate responses. Nature medicine. 2007;13 10):124852.

24. Palm NW, Medzhitov R. Not so fast: adaptive suppression of innate immunity. Nature medicine. 2007;13(10):1142-4.

25. Cecere TE, Todd SM, Leroith T. Regulatory $\mathrm{T}$ cells in arterivirus and coronavirus infections: do they protect against disease or enhance it? Viruses. 2012;4(5):833-46.

26. Maloir Q, Ghysen K, von Frenckell C, Louis R, Guiot J. [Acute respiratory distress revealing antisynthetase syndrome]. Rev Med Liege. 2018;73(7-8):370-375.

27. Chu CM, Cheng VC, Hung IF, et al. Role of lopinavir/ itonavir in the treatment of SARS: initial virological and clinical findings. Thorax. 2004;59(3):252-6.

28. Holshue ML, DeBolt C, Lindquist S, et al. First Case of 2019 Novel Coronavirus in the United States. N Engl J Med. 2020.[doi:10.1056/NEJMoa2001191]

29. Sheahan TP, Sims AC, Leist SR, et al. Comparative therapeutic efficacy of remdesivir and combination lopinavir, ritonavir, and interferon beta against MERS-CoV. Nature communications. 2020;11(1):222.

30. Russell CD, Millar JE, Baillie JK. Clinical evidence does not support corticosteroid treatment for 2019-nCoV lung injury. Lancet. 2020;395(10223):473-475. 